\title{
N-P Transition Sensing Response of Strontium Titanate Ferrite Sol-Gel Thin Films
}

\author{
Chee Lap Chow ${ }^{1}$, Ooi Kiang Tan ${ }^{1}$, Man Siu Tse ${ }^{1}$ \\ ${ }^{1}$ School of Electrical and Electronic Engineering, \\ Nanyang Technological University, \\ 50 Nanyang Avenue, \\ 639798 Singapore \\ Email: eoktan@ntu.edu.sg
}

\begin{abstract}
:
Perovskite oxide has been reported as a potential gas sensing material in addition to commonly used binary oxide. Strontium titanate ferrite $\left(\mathrm{SrTi}_{0.6} \mathrm{Fe}_{0.4} \mathrm{O}_{3}\right)$ thin film sensors were fabricated using modified sol-gel spin coating technique and annealed at different annealing temperatures. An anomalous $n-$ to p-type transition of oxygen sensing response was observed as annealing temperature increased. A typical p-type sensing response was observed for sensors annealed at temperature of $500{ }^{\circ} \mathrm{C}$ and higher. On the other hand, sensors annealed below $450{ }^{\circ} \mathrm{C}$ showed an unusual $\mathrm{n}$-type sensing response, which has never been reported. The n-type sensing response was caused by the amorphous phases in the thin film annealed at low annealing temperature, as revealed by X-ray diffraction (XRD), tunneling electron microscopy (TEM) and X-ray photoelectron spectroscopy (XPS).
\end{abstract}

Key words: strontium titanate ferrite, gas sensors, oxygen sensors, p-n transition.

\section{Introduction}

Gas sensor technology is one of the most important key technologies of the future development with a constantly increasing number of applications in environmental monitoring, pollution control, healthcare, automobiles, hydrogen economy and technical processes control. Since the discovery of semiconductor metal oxide gas sensors in 1962, most of the research works on were focused on relatively simple binary oxides such as $\mathrm{SnO}_{2}, \mathrm{ZnO}$ and $\mathrm{TiO}_{2}$.

Additionally, perovskite oxides with $\mathrm{ABO}_{3}$ structure was also reported to be a promising gas sensing material due to its attractive properties such as excellent gas sensitivity, mixed ionic-electronic conductivity, great doping flexibility, ability to accommodate large amount of dopants and defects, high melting and decomposition temperature [1,2]. Particularly, strontium titanate ferrite (STFx) appeared as a potential candidate for oxygen sensor. In strontium titanate ferrite solid solution system, trivalent iron ion $\left(\mathrm{Fe}^{3+}\right)$ substitutes for the quadrivalent titanium ion $\left(\mathrm{Ti}^{4+}\right)$ without restoring the electroneutrality in the cation site. Due to the difference in their preferential oxidation states, oxygen vacancies are formed to maintain charge balance in the system [3]. This inherent non-stoichiometry makes STFx highly sensitive to oxygen partial pressure.

In this study, STF40 $(x=0.4)$ thin films were deposited using modified sol-gel spin-coating technique. To our knowledge, the n- to p-type transition of sensing response was observed for the first time for STFx sol-gel thin film sensors. The underlying mechanism for this phenomenon was investigated with the help of various characterization techniques.

\section{Experimental Procedures}

For the preparation of STF40 sol-gel precursor, titanium butoxide (Aldrich, 97\%) was first thoroughly mixed with acetylacetone (Merck, $99 \%$ ) with a mole ratio of $1: 4$. Then, iron nitrate (Merck, >99\%) was dissolved in 2methoxyethanol (Sigma-Aldrich, 99.8\%) and acetylacetone with a mole ratio of 1:20:6. Next, the $\mathrm{Ti}+\mathrm{Fe}$ precursor was mixed together and homogenized under continuous stirring. On the other hand, the strontium acetate (NacalaiTesque) was dissolved in glacial acetic acid and deionized water with a mole ratio of 1:15:90. Finally, the dissolved $\mathrm{Sr}$ precursor solution was dripped into the $\mathrm{Ti}+\mathrm{Fe}$ precursor with continuous stirring using a magnetic stirrer. The viscosity and the molarity of the final sol were adjusted using 2-methoxyethanol. The STF40 sol was spun onto the $\mathrm{Si} / \mathrm{SiO}_{2}$ wafer 
substrate with gold interdigitated electrodes at $3000 \mathrm{rpm}$ for 30 seconds. The soft bake temperatures were set as $150^{\circ} \mathrm{C}$ and $300^{\circ} \mathrm{C}$ for 5 minutes each. Then, the spin-coating cycle was repeated for five times to obtain multiplelayer thin films sensors. The final films were annealed at different temperatures for one hour under air ambient with flow rate of $1 \mathrm{slm}$ using Lindberd tube furnace.

The XRD patterns were recorded at room temperature using Siemens D5005 X-ray diffractometer with $\mathrm{Cu} \mathrm{K}$-alpha radiation $(\lambda=$ $1.5406 \AA$ ) operating at $40 \mathrm{kV}$ and $40 \mathrm{~mA}$. The surface morphologies of the thin films were observed using FESEM (Jeol-6340F). The microstructure of the thin films was analyzed using a TEM (JEOL 2010) operating at 200 $\mathrm{kV}$.XPS measurement was carried out using a Kratos AXIS spectrometer with monochromatic Al Ka (225W, $15.0 \mathrm{kV}$ with kinetic energy of $1486.6 \mathrm{eV}) \mathrm{X}$-ray radiation. The calibration of the binding energy scale was performed with the ubiquitous carbon contamination peak located at $285 \mathrm{eV}$. The oxygen sensing properties were characterized using a customdesigned gas sensing characterization system.

\section{Results and Discussion}

The structural development in sol-gel derived STF40 thin films with different annealing temperatures was studied using XRD and their XRD patterns are illustrated in Fig. 1. For asdeposited film and film annealed at $400{ }^{\circ} \mathrm{C}$, no significant phase was observed and the films were still in amorphous phase. With increasing of the annealing temperature, the cubic perovskite phase was slowly developed. One can observe that the relative intensity of STF40 cubic perovskite peaks increased with annealing temperature, indicating better crystallinity of the thin film.

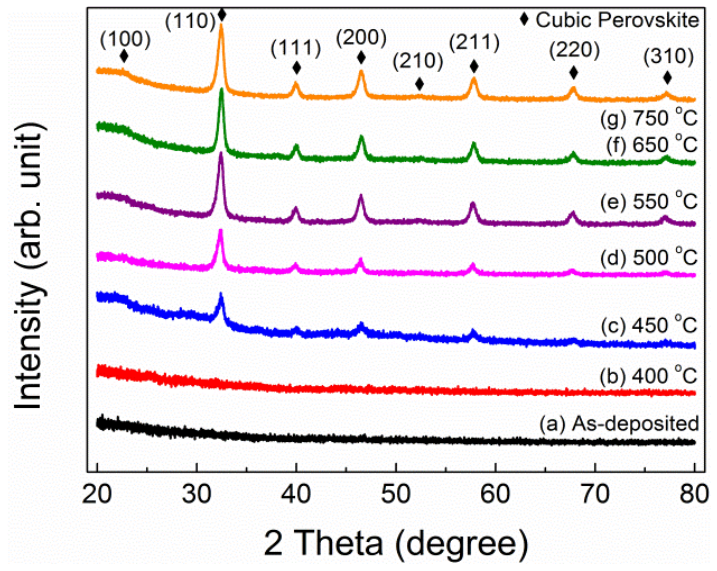

Fig. 1. XRD patterns of the STF40 thin films annealed at different temperatures for $1 \mathrm{~h}$.
The oxygen gas sensing property of the STF40 thin film sensors was characterized at different operating temperatures by measuring the changes in electrical resistance upon exposure of $20 \%$ oxygen gas and the results are plotted in Fig. 2. For sensors annealed at 400 and 450 ${ }^{\circ} \mathrm{C}$, an increase in the electrical resistance was observed upon oxygen gas exposure, as shown in Fig. 2(a). At low operating temperatures (150 to $\left.300{ }^{\circ} \mathrm{C}\right)$, the bulk equilibration with oxygen is kinetically hindered and is a very slow process. Hence, the oxygen sensing property is mainly contributed from the surface oxygen chemisorption which causes formation of oxygen adsorbates on the grain surface. In the case of n-type semiconductor metal oxide, oxygen chemisorption extracts electrons from the bulk and builds a space-charge region on the grain surface [4]. Formation of spacecharge region causes a decrease in the major carriers that lead to an increase of electrical resistance. On the other hand, sensors annealed at $\geq 550{ }^{\circ} \mathrm{C}$ exhibited a normal p-type sensing response manifested by a decrease in resistance upon oxygen gas exposure, as shown in Fig. 2(b), which is in good agreement with reported works on STFx sensors $[5,6]$.
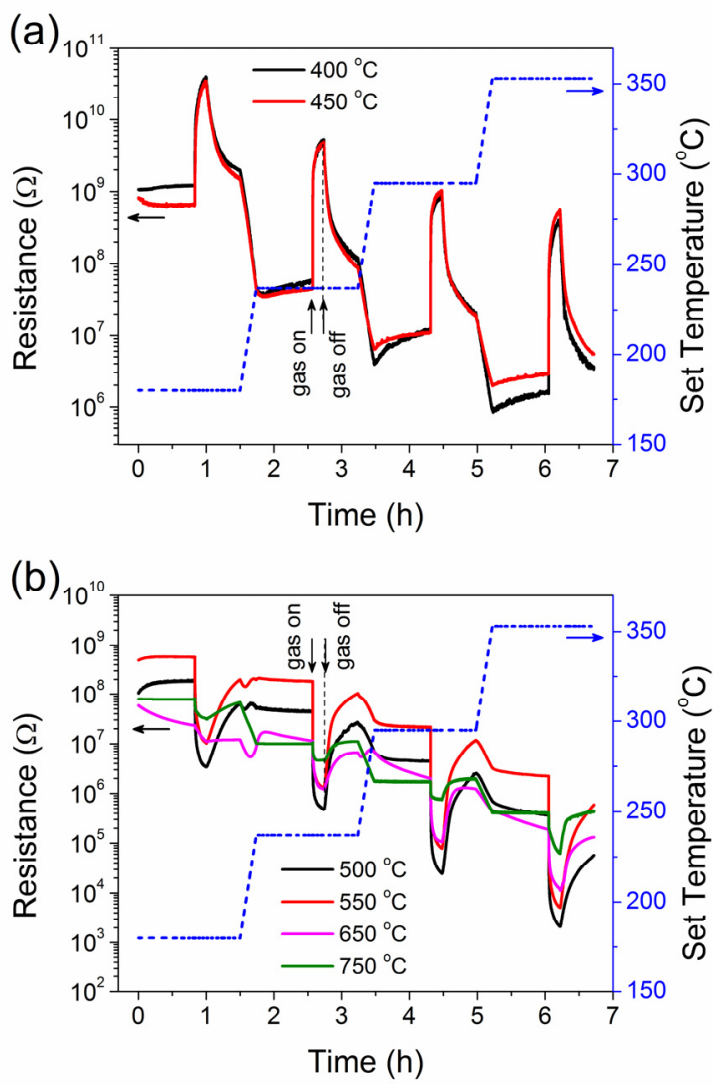

Fig. 2. Gas sensing responses to $20 \%$ oxygen in nitrogenat different operating temperatures for STF40 sol-gel thin film sensors annealed at different temperatures: (a) n-type and (b) p-type sensing response. 
The n-type sensing response of the STF40 film sensors has never been reported and it is worth for in-depth study. Generally, semiconducting metal oxide sensors are usually classified as "ntype" sensors and "p-type" sensors. The sensing response depends on the surface conductivity type of the metal oxides, which is determined by the majority electronic carriers at the surface, for example electrons or holes. However, transition of the sensing response of the oxides has also been observed and reported [7-10]. The explanation of the switching of sensing response type is still a matter of debate and various mechanisms have been adopted to explain this phenomenon.

In our case, the $n-p$ transition was trigged by the annealing temperature of the STF40 film sensor, so we believed that the phase structure of the thin films resulted in this $n-p$ transition. Next, the microstructure of the STF40 thin films was further characterized using HRTEM. Fig. 3 shows the high resolution TEM image for STF40 thin films annealed at 450 and $650{ }^{\circ} \mathrm{C}$. The microstructure of thin film annealed at 450 ${ }^{\circ} \mathrm{C}$ had an amorphous-like morphology with initial crystallization. This indicates that the thin film was still in amorphous phase and starting to form cubic perovskite phase. On the other hand, the film annealed at $650{ }^{\circ} \mathrm{C}$ had polycrystalline phase and tiny nanocrystals with diameter about $3 \mathrm{~nm}$ are clearly visible in Fig. 3(b). The TEM observations are certainly in good agreement with the XRD measurements.

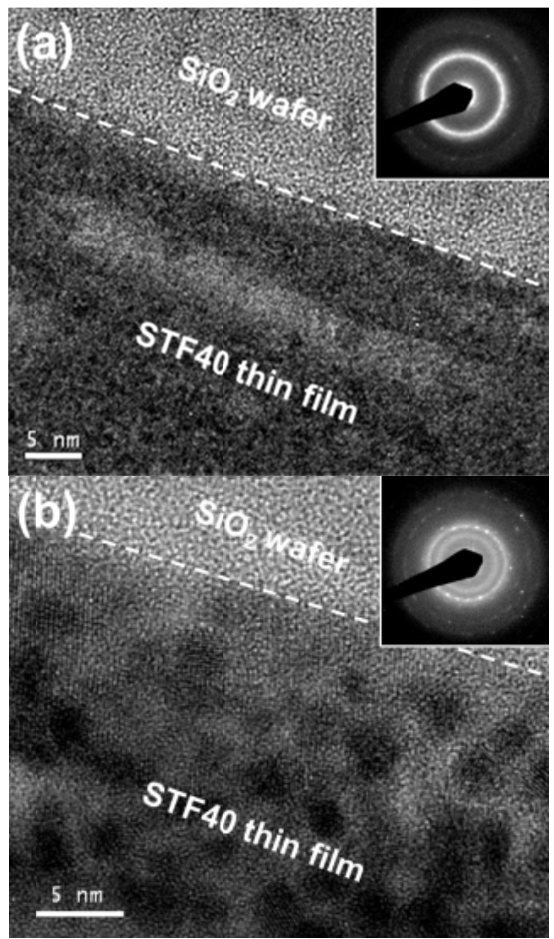

Fig. 3. HRTEM and SAED patterns for STF40 solgel thin films annealed at (a) $450{ }^{\circ} \mathrm{C}$ and (b) $650{ }^{\circ} \mathrm{C}$ for $1 h$.
Next, the surface chemical state in STF40 thin films with different annealing temperatures was studied using XPS. It's is remarkable to observe two different pairs of $\mathrm{Ti} 2 \mathrm{p}$ doublet peaks from the XPS spectra, labeled as doublet peaks Ti2p $A$ and $\mathrm{Ti} 2 \mathrm{p} \mathrm{B}$, as depicted in Fig. 4(a). The doublet peaks $B$ at higher binding energy $(459.2 \mathrm{eV})$ is similar to the $\mathrm{Ti}^{4+}$ peaks of the commercial rutile $\mathrm{TiO}_{2}$ which was detected in another independent XPS experiment. On the other hand, the doublet peaks $A$ located at slightly lower binding energy $(458.0 \mathrm{eV})$ was assigned as the $\mathrm{Ti}^{4+}$ in the cubic perovskite structure $[11,12]$. Only Ti2p B was detected in the film annealed at $400{ }^{\circ} \mathrm{C}$, showing that the element $\mathrm{Ti}$ was in the $\mathrm{TiO}_{2}$-like bonding. At annealing temperatures of 450 and $550{ }^{\circ} \mathrm{C}$, this peaks $B$ co-existed with peaks $A$, indicating that the $\mathrm{Ti}$ was bonded in two different types of bonding i.e. $\mathrm{Ti}-\mathrm{O}$ bond in $\mathrm{TiO}_{2}$ structure and $\mathrm{Ti}-$ $\mathrm{O}$ bond in perovskite structure. For films annealed at higher annealing temperatures (650 and $750{ }^{\circ} \mathrm{C}$ ), only doublet peaks Ti2p A existed indicating complete formation of cubic perovskite phase.

As illustrated in Fig. 4(b), O1s XPS spectra mainly consist of three peaks, namely O1s $A$ (529.3 eV), O1s B ( 531.3 eV), and O1s C $(\sim 532.6 \mathrm{eV})$. The O1s A peak with lowest binding energy was assigned as the doublecharged lattice oxygen $\left(\mathrm{O}^{2-}\right)$ in the cubic perovskite structure. We can observe that the lattice oxygen was only existed for films annealed at $450{ }^{\circ} \mathrm{C}$ and higher. The $\mathrm{O} 1 \mathrm{~s}$ lateral oxygen peaks located at the higher binding energy range corresponds to the ionizations of weakly adsorbed species or excess oxygen on the surface. Specifically, O1s B and O1s C were assigned to surface carbonate species and absorbed molecular oxygen species, respectively.
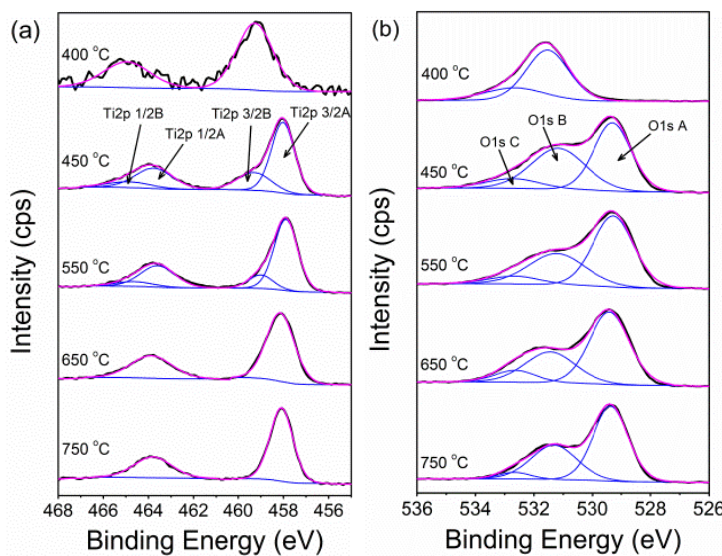

Fig. 4. XPS high resolution spectra for STF40 solgel thin film sensors annealed at different temperatures: (a) Ti2p and (b) O1s. 
As a summary, lower annealing temperature ( $\leq$ $450{ }^{\circ} \mathrm{C}$ ) is insufficient to completely form the $\mathrm{p}$ type cubic perovskite phase for STF40 sol-gel thin film. The films mainly contain the amorphous phases such as $\mathrm{TiO}_{2}$ and $\mathrm{Fe}_{2} \mathrm{O}_{3}$ which formed by the decomposition of the solgel precursor. Unfortunately, due to the asymmetry and broadening of the Fe2p XPS spectra, we could not obtain any significant information about structure change as annealing temperature increased. Hence, the ntype sensing response observed in the STF40 film sensor annealed at temperature below 450 ${ }^{\circ} \mathrm{C}$ was resulted from these n-type amorphous phases. At higher annealing temperatures, the STF40 film crystallized well into p-type cubic perovskite phase and the sensors showed normal $p$-type sensing response.

\section{Conclusion}

STF40 sensors were deposited using a modified sol-gel spin-coating method and annealed at 400 to $750{ }^{\circ} \mathrm{C}$ for 1 hour. According to the XRD study, the peaks intensity and hence the crystallinity of the cubic perovskite phase increased with annealing temperature. Oxygen sensing results showed $n$ - to p-type oxygen sensing response transition as the annealing temperature increased. In contrast to typical p-type sensing response for STF40 sensors annealed at $500{ }^{\circ} \mathrm{C}$ and higher temperatures, an anomalous n-type sensing response was observed for sensors annealed at $400{ }^{\circ} \mathrm{C}$ and $450{ }^{\circ} \mathrm{C}$. This phenomenon was further investigated using TEM and XPS. TEM observation revealed that the film annealed at $450{ }^{\circ} \mathrm{C}$ was still in amorphous phase with poor crystallization while the film annealed at $650{ }^{\circ} \mathrm{C}$ showed polycrystalline structure. In addition, the existence of amorphous $\mathrm{TiO}_{2}$ phase and lattice oxygen in the films was also witnessed using XPS. Hence, the n-type sensing response was attributed to the contribution of amorphous $\mathrm{TiO}_{2}$ and $\mathrm{Fe}_{2} \mathrm{O}_{3}$ phase in the films annealed at lower temperature.

\section{References}

[1] R. Moos, N. Izu, F. Rettig, S. Reiß, W. Shin, and I. Matsubara, "Resistive oxygen Gas Sensors for Harsh Environments," Sensors 11, 3439-3465 (2011); doi: 10.3390/s110403439

[2] J. W. Fergus, "Perovskite oxides for semiconductor-based gas sensors," Sensors and Actuators B: Chemical 123, 1169-1179 (2007); doi: 10.1016/j.snb.2006.10.051

[3] A. Rothschild, W. Menesklou, H. L. Tuller, and E. Ivers-Tiffee, "Electronic structure, defect chemistry, and transport properties of $\mathrm{SrTi}_{1}$ ${ }_{x} \mathrm{Fe}_{\mathrm{x}} \mathrm{O}_{3-\mathrm{y}}$ solid solutions," Chemistry of Material 18, 3651-3659 (2006); doi: 10.1021/cm052803x
[4] P. T. Moseley, "New trends and future prospects of thick- and thin-film gas sensors," Sensors and Actuators B: Chemical 3, 167-174 (1991); doi: 10.1016/0925-4005(91)80002-2

[5] K. Sahner, R. Moos, M. Matam, J. J. Tunney, and M. Post, "Hydrocarbon sensing with thick and thin film p-type conducting perovskite materials," Sensors and Actuators B: Chemical 108, 102-112 (2005); doi: 10.1016/j.snb.2004.12.104

[6] K. Sahner, R. Moos, M. Matam, and M. Post, "Thick and thin film p-type conducting perovskite hydrocarbon sensors - a comparative study," Proceedings of IEEE Sensors 2, 926-931 (2003); doi: 10.1109/ICSENS.2003.1279078

[7] A. Gurlo, M. Sahm, A. Oprea, N. Barsan, and U. Weimar, "A p- to n-transition on $\alpha-\mathrm{Fe}_{2} \mathrm{O}_{3}$-based thick film sensors studied by conductance and work function change measurements," Sensors and Actuators B: Chemical 102, 291-298 (2004); doi: 10.1016/j.snb.2004.04.075

[8] G. Korotcenkov, V. Brinzari, V. Golovanov, A. Cerneavschi, V. Matolin, and A. Tadd, "Acceptorlike behavior of reducing gases on the surface of n-type $\operatorname{In}_{2} \mathrm{O}_{3}$," Applied Surface Science 227, 122131 (2004); doi: 10.1016/j.apsusc.2003.11.051

[9] M. Sánchez, M. E. Rincón, and R. A. GuiradoLópez, "Anomalous sensor response of $\mathrm{TiO}_{2}$ films: Electrochemical impedance spectroscopy and ab Initio studies," Journal of Physical Chemistry C 113, 21635-21641 (2009); doi: 10.1021/jp9024014

[10] H. Huang, H. Gong, C. L. Chow, J. Guo, T. J. White, M. S. Tse, and O. K. Tan, "Lowtemperature growth of $\mathrm{SnO}_{2}$ nanorod arrays and tunable $n-p-n$ sensing response of a $\mathrm{ZnO} / \mathrm{SnO}_{2}$ heterojunction for exclusive hydrogen sensors," Advanced Functional Materials 21, 2680-2686 (2011); 10.1002/adfm.201002115

[11] A. Kosola, M. Putkonen, L.-S. Johansson, and L. Niinisto, "Effect of annealing in processing of strontium titanate thin films by ALD," Applied Surface Science 211, 102-112 (2003); doi: 10.1016/S0169-4332(03)00175-2

[12] M. Murata, K. Wakino, and S. Ikeda, "X-ray photoelectron spectroscopic study of perovskite titanates and related compounds: an example of the effect of polarization on chemical shifts," Journal of Electron Spectroscopy and Related Phenomena 6, 459-464 (1975); doi: $10.1016 / 0368-2048(75) 80032-6$ 